\title{
Omega plate for the treatment of acetabular fractures involving the quadrilateral plate
}

\author{
BOGDAN VELICEASA ${ }^{1}$, ALEXANDRU FILIP ${ }^{1}$, MIHAELA PERTEA ${ }^{2}$, \\ DRAGOS POPESCU ${ }^{1}$, CLAUDIU CARP $^{1}$ and OVIDIU ALEXA ${ }^{1}$ \\ ${ }^{1}$ Department of Orthopedics and Traumatology, Surgical Sciences (II), ${ }^{2}$ Department of Plastic Surgery, Surgical Sciences (I), \\ 'Grigore T. Popa' University of Medicine and Pharmacy, Faculty of Medicine, 700115 Iasi, Romania
}

Received May 24, 2021; Accepted June 23, 2021

DOI: $10.3892 /$ etm.2021.10498

\begin{abstract}
This retrospective study aimed to assess the outcome of a modified Stoppa approach using an anatomically precontoured plate for the treatment of acetabular fractures. In total, 30 patients (mean age 50.3 years; 25 men and 5 women) with acetabular fractures were treated between January 1, 2018 and December 31, 2019. In all 30 cases, fracture reduction was performed through a modified Stoppa approach and fixed with the omega plate. In specific fracture patterns, additional approaches were needed (lateral window in 4 cases and posterior Kocher-Langenbeck approach in 7 cases). Patients were assessed for restoration of the hip joint congruency, complications, and overall fracture reduction. Quality of reduction was categorized based on Matta's radiological principles and to assess functional outcome the Merle d'Aubigné-Postel and Harris hip score was used. The average anesthesia time was $253.6 \mathrm{~min}$, the mean intraoperative blood lost was $266.6 \mathrm{ml}$ and the mean intraoperative fluoroscopy dose was $3.21 \mathrm{mGy}$. According to Matta criteria for reduction quality, anatomical reduction was recorded in 22 cases, imperfect reduction in 6 cases and 2 cases had poor reduction. The average follow-up was 22.5 months. Malunion, loss of reduction or implant loosening were not recorded. Late complications included one case of avascular necrosis of the femoral head and post-traumatic arthritis changes in 5 cases. At the final follow-up, a mean Merle d'Aubigné-Postel score of $13.26 \pm 4.46$ and a mean Harris score of $86.03 \pm 13.37$ were recorded. The possibility
\end{abstract}

Correspondence to: Dr Alexandru Filip, Department of Orthopedics and Traumatology, Surgical Sciences (II), 'Grigore T. Popa' University of Medicine and Pharmacy, Faculty of Medicine, 16 Universității Street, 700115 Iasi, Romania

E-mail: alexandru.filip@ymail.com

Dr Mihaela Pertea, Department of Plastic Surgery, Surgical Sciences (I), 'Grigore T. Popa' University of Medicine and Pharmacy, Faculty of Medicine, 16 Universității Street, 700115 Iasi, Romania E-mail: pertea_mihaela@yahoo.com

Key words: fixation, trauma, acetabular fracture, omega plate, treatment of an anatomically precontoured plate with subsequent lower operative time combined with stable fixation of the primary acetabular fracture fragments and the quadrilateral plate makes the omega plate a viable option for treating acetabular fractures with a very low complication rate and good to excellent results in $89 \%$ of the cases.

\section{Introduction}

Acetabular fractures are intra-articular fractures, in general due to high energy trauma, and are frequently associated with major organ injuries. Consequently, they remain a major challenge for the orthopedic surgeon. The gold standard for the treatment of displaced and unstable acetabular fractures is surgery. Only anatomic reduction with stable fixation and early rehabilitation lead to optimal results (1). Several approaches are available such as the Kocher-Langenbeck (K-L), iliofemoral, ilioinguinal, combined anterior and posterior approaches, extended iliofemoral, transtrochanteric, and triradiate approaches (2). Each of these approaches have their own advantages depending on the fracture pattern, but despite the improvements in operative treatment, the complication rate remains high leading to poor long-term outcome $(3,4)$.

Since it was initially described by Letournel in 1961, the ilioinguinal approach has been the only intrapelvic approach employed (5). The limitation of the approach is that it offers a limited view to the quadrilateral plate, lack of direct visualization of the acetabular surface and control in severely displaced posterior column fractures. In addition, a high rate of postoperative infection and iatrogenic injury to the ilio-femoral vessels and femoral nerve is common (6-9).

Over the years, changes have been made to the ilioinguinal approach to reduce morbidity. In 1994, Cole and Bolhofner described the modified Stoppa approach as an alternative to the ilioinguinal approach as causing least tissue damage (10). The most important difference between the two approaches is that the modified Stoppa approach avoids the 'middle window', thereby having less incidence of damage to the ilio-femoral vessels and femoral nerve. Additionally, it offers a good view of the quadrilateral plate and the medial aspect of the posterior column. However, it does not offer good exposure to the iliac wing, and for this reason, especially in high anterior column 
fractures, additional lateral (iliac wing) window opening is necessary $(11,12)$.

Usually, reduction and stabilization of the quadrilateral plate fragment is challenging. Different types of implants have been developed over time, and, among these, anatomical plates that stabilize both the primary fracture and quadrilateral plate fragment have become more popular.

In this study, we aimed to assess the outcome of a modified Stoppa approach using an anatomically precontoured plate in the treatment of acetabular fractures.

\section{Patients and methods}

Patient data. From January 1, 2018 to December 31, 2019, 61 consecutive patients with acetabular fractures were treated at the Trauma Department of the University Hospital, Iasi, Romania. From these 30 patients, 25 were men (83.3\%) and 5 were women with a mean patient age of $50.36 \pm 14.47$ years (range from 19 to 73 years). Inclusion criteria were patients $>18$ years of age, diagnosis of displaced acetabular fractures with involvement of the quadrilateral plate, and patients suitable for surgical treatment. Exclusion criteria were refusal to give informed consent, acetabular fracture which did not require reduction and fixation of the quadrilateral plate (anterior wall, posterior column and/or posterior wall fractures). The 30 patients underwent open reduction and internal fixation (ORIF) of the acetabular fracture via the modified Stoppa approach. In these cases, final fixation of the fracture was performed using the omega plate (Medin, Nové Město na Moravě, Czech Republic).

At admission, plain X-ray, antero-posterior (AP) view of the pelvis and computed tomography (CT) scan were taken for all the patients in the emergency room to assess fracture patterns and for preoperative planning. Radiologic images were independently assessed for fracture classification and parameter measurements by two orthopedic specialists and one fellow orthopedic resident using RadiAnt DICOM Viewer (Medixant).

Fracture patterns were assessed according to the Judet and Letournel classification (2). Femoral head subluxation and/or dislocation, intra-articular free fragments, comminution and/or impaction of the acetabular surface, articular surface gap and/or step-off were assessed in each case prior to surgery.

All of the surgical procedures were performed by the same senior surgeon. All the patients were carefully informed regarding the potential risks and benefits of the intervention, and their informed consent was obtained (13).

Informed consent was obtained from all the individual participants included in the study. The study was conducted according to the Helsinki Declaration and was approved by the Ethics Committee of the 'Sf. Spiridon' Emergency Hospital Iasi, Romania.

Preoperative planning. The omega plate was precontoured preoperatively according to the patient CT scan images. Based on the CT scan, the projection of the pelvic inlet (CTD) was traced as described by Taller et al. The inclination of the cut on sagittal plane was determined by a line which connects the ventral upper edge of the symphysis and sacrum, $10 \mathrm{~mm}$ below the promontorium (14). Then, on the coronal CT view, the line was moved caudally, symmetrically on both sides, to be centred in the cranial $1 / 4$ of the femoral heads. In this manner we obtained, on the axial CT view, the full contour of the pelvic brim. The plate arch was determined according to the uninjured side. The plate centre should match the acetabular mid-point, dorsal part of the plate should not cross the sacroiliac joint and the ventral part ends in the proximity of the pubic symphysis. Then the plate hitches were bent: The ventral ones at $80^{\circ}$, the compression ones at $75^{\circ}$ and the dorsal ones $60^{\circ}$ in men and $50^{\circ}$ in women (14) (Fig. 1).

Surgical technique. The patients were positioned supine on the radiolucent operating table. Fracture reduction was assessed fluoroscopically with $\mathrm{C}$-arm. The lower abdomen and the entire lower limb were draped aseptically. A sandbag was placed under the knee to maintain the hip flex and to relax the iliopsoas muscle and the iliac neurovascular bundle. A midline incision of $12-15 \mathrm{~cm}$ was performed proximal to the pubic symphysis. The abdominal fascia was exposed and the linea alba was identified. After the incision of the linea alba, the rectus abdominis muscle was divided, and the bladder underneath. On approaching the inside of the pelvis, blunt dissection of the Retzius space was performed, the pubic symphysis was identified, the dissection was continued, subperiosteally, at the level of the pubic ramus. Laterally, the corona mortis was identified and ligated. The subperiosteal dissection was performed along the pelvis brim to the sacro-iliac joint. The obturator neurovascular bundle was identified and protected. Extreme caution was taken when placing the Hohman retractor underneath the iliopsoas muscle to protect the external iliac artery and vein. After the fracture site exposure, the reduction was attempted, followed by the internal fixation with an omega plate. The pre-contoured plate was applied just caudally of the arcuate line and temporally fixed anteriorly with a K-wire inserted through the most ventral hitch into the pubis. Plate position and contouring were then checked, and if necessary, additional plate contouring or hitch bending were performed. The K-wire was then replaced with a long symphyseal screw, and using a pelvic ball-spike, and the plate was pressed against the quadrilateral plate. Additionally, with a second pelvic ball-spike, pressing on the compression hitches, the bone was pressed above the arcuate line. In this manner, final realignment of the pelvic arch was obtained. Subsequently, a proximal screw was placed throughout a proximal hole. Additional screws were placed depending on the fracture pattern.

In cases where a modified Stoppa approach alone was insufficient for fracture open reduction and internal fixation (e.g., high anterior column fracture), an additional lateral window was performed along the iliac crest, and, in fractures in which the reduction could not be performed only with the anterior approach (e.g., associated posterior wall fracture), a second concurrently posterior Kocher-Langenbeck (K-L) approach was performed.

After treatment and follow-up. Only one dose of antibiotics (third generation cephalosporin) was used intraoperatively. Prophylaxis for thromboembolism was started during admission using low molecular weight heparin and continued for 35 days postoperatively. Indomethacin was administered for 

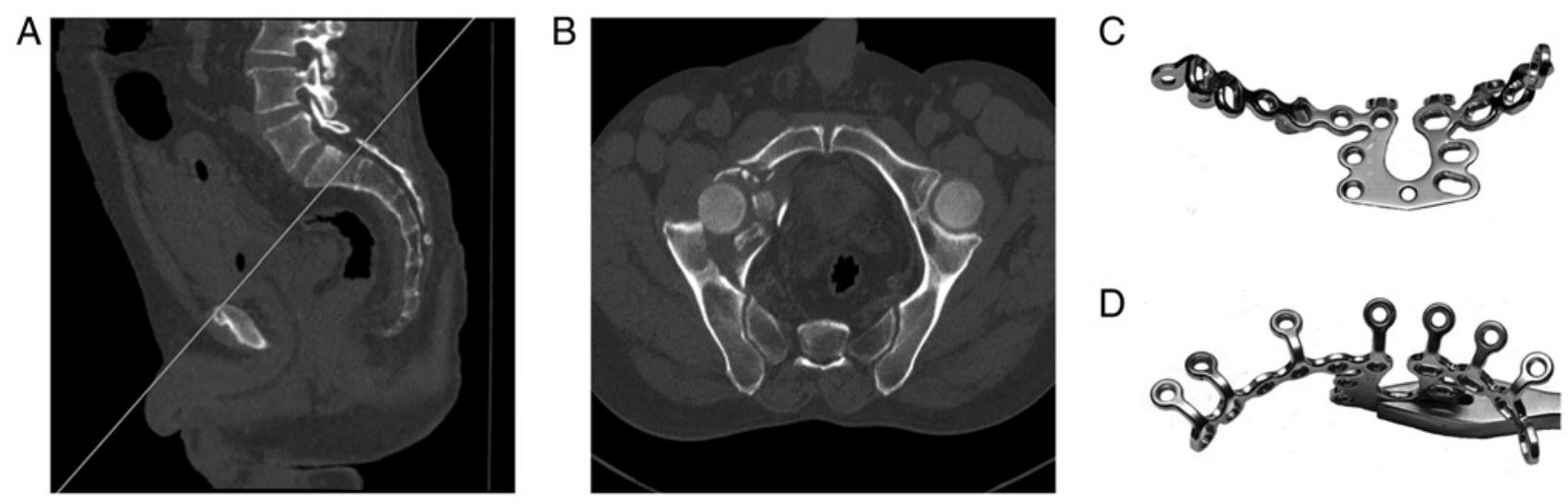

Figure 1. Example of the preoperative planning for the treatment of an acetabular fracture with an omega plate. (A) First the inclination of the cut for the projection of the pelvic inlet on the sagittal CT scan views was traced. (B) The second step was to center the plane in the cranial $1 / 4$ of the femoral head on the axial CT scan views. The heart shape of the pelvic inlet, particularly in males, on this image was observed; on the opposite end, the shape for the plate was traced. Precontoured omega plate according to the CT scan images [(C) medial view, (D) superior view].

heterotopic ossification prevention for 2 weeks in patients with combined approaches. Drainage was removed 24-48 h after surgery. Physical therapy was started the second day after surgery with early out-of-bed mobilization and active and passive range of motions (ROM). Weight bearing was not allowed for the first six weeks postoperatively, followed by rapidly progressive weight bearing to full weight bearing at 10-12 weeks.

Patients were assessed for restoration of the hip joint congruency, complications, and overall fracture reduction on plain X-ray (AP, obturator and iliac views) and CT scans. Quality of reduction was categorized based on Matta's radiological principles: Reduction was considered anatomical if all five anatomical lines were restored, all fracture gaps and/or steps had been removed and the femoral head was centered under the acetabular roof $(1,15)$.

Postoperative clinical and radiological assessment was performed at 6 weeks, 3, 6 and 12 months and after 12 months using Merle d'Aubigné-Postel and Harris hip score and Matta Criteria (1,15-17).

Statistical analysis. Data were analyzed using SPSS v18.0 software (IBM Corp.). Descriptive statistics and frequency analysis were performed for all parameters. The following statistical tests were performed: Repeated measures ANOVA test post hoc Bonferroni correction, the unpaired Student's t-test, $\chi^{2}$ test, and Pearson correlation. The significance level was set a priori at $\mathrm{P}<0.05$.

The inter-observability was measured using Cohen's $\kappa$ coefficient $(\mathrm{k})$. We obtained a $\mathrm{k}=0.90$ (almost perfect agreement) for fracture classification, and $\mathrm{k}=0.74$ (substantial agreement) for other parameters measured. No intra-observer reliability was performed.

\section{Results}

Demographic characteristics of the patients. The mean patient age was $50.36 \pm 14.47$ years. Twenty-five patients $(83.3 \%)$ were men and 5 patients were women (male/female=5/1). Among the 30 acetabular fractures, the left side was fractured in 18 cases $(60 \%)$ (Table I). All acetabular fractures were due to high energy trauma: Falls from a height $(50 \%)$ or car accidents (50\%).

According to the Judet and Letournel (2) classification, six $(20 \%)$ fractures were categorized as anterior column (AC), one $(3.3 \%)$ as pure transverse (PT), seven $(23.3 \%)$ as transverse + posterior wall (TPW), eight (26.7\%) as anterior column + posterior hemitransverse (ACPhT) and eight $(26.7 \%)$ as both column (BC). Additionally, 19 (63.3\%) patients suffered other accompanying injuries, among these, most common were fracture in other areas (12 patients) and thorax injuries (11 patients).

All the associated femoral head dislocations were reduced in emergency, and the acetabular fractures were surgically treated on average 4.53 days after injury (range: 2-10 days). In $19(63.3 \%)$ patients the modified Stoppa approach alone offered enough exposure for ORIF. Among these 19 patients, bilateral Stoppa approach was performed in one case. In $4(13.3 \%)$ patients, additional opening of the lateral window was required, in high anterior column fractures, and $7(23.3 \%)$ patients required concomitant posterior approach (Kocher-Langenbeck approach), i.e., those with posterior wall fracture association. The anesthesia time was on average $253.6 \mathrm{~min}$ (range: 136-465 $\mathrm{min}$ ). The mean intraoperative blood lost was determined to be $266.6 \mathrm{ml}$ (range: $130-996 \mathrm{ml}$ ) and mean intraoperative blood transfusion was 1.1 units (range: 0-3 units). The mean intraoperative fluoroscopy dose was $3.21 \mathrm{mGy}$ (range: 1.01-7.02 mGy).

Surgical technique. Intraoperatively, one patient had an obturator vein injury, while another patient had both obturator and external iliac vein injury. In both cases, the vascular lesions were repaired by the cardiovascular surgeon. Postoperatively, the two patients developed superficial wound infection which was successfully treated within 1-2 weeks with debridement, antibiotic therapy and vacuum-assisted closure (VAC). One patient developed lateral abdominal wall hernias at 6 months after surgery, associated with lateral window approach, which was repaired by the general surgeon. No obturator or lateral femoral cutaneous nerve palsy was recorded.

Postoperatively, according to the Matta criteria for reduction quality, anatomical reduction in 22 cases, imperfect 
Table I. Demographic characteristics of the patients.

\begin{tabular}{|c|c|}
\hline Characteristics & Data values \\
\hline Mean age (years) & $50.36 \pm 14.47$ \\
\hline $\operatorname{Sex}(M / F)$ & $25 / 5$ \\
\hline Side $(\mathrm{L} / \mathrm{R})$ & $18 / 12$ \\
\hline \multicolumn{2}{|l|}{ Injury, n (\%) } \\
\hline RTA & $15(50)$ \\
\hline Fall from height & $15(50)$ \\
\hline \multicolumn{2}{|l|}{ Fracture type, n (\%) } \\
\hline Anterior column & $6(20)$ \\
\hline Pure transverse & $1(3.3)$ \\
\hline Transverse + posterior wall & $7(23.3)$ \\
\hline Anterior column + posterior hemitransverse & $8(26.7)$ \\
\hline Both columns & $8(26.7)$ \\
\hline \multicolumn{2}{|l|}{ Femoral head dislocation, $\mathrm{n}(\%)$} \\
\hline Posterior & $5(16.6)$ \\
\hline Medial & $10(33.3)$ \\
\hline \multicolumn{2}{|l|}{ Femoral head surface, n (\%) } \\
\hline Chondral lesion & $8(26.6)$ \\
\hline Intra-articular fragments & $8(26.6)$ \\
\hline Fracture & $1(3.3)$ \\
\hline \multicolumn{2}{|l|}{ Acetabular surface, n (\%) } \\
\hline Chondral lesion & $12(40)$ \\
\hline Impacted fracture & $9(30)$ \\
\hline \multicolumn{2}{|l|}{ Associated injury, n (\%) } \\
\hline Thorax & 11 \\
\hline Abdomen & 6 \\
\hline Genitourinary tract & 2 \\
\hline Extremities & 12 \\
\hline Time from trauma to surgery (days) & $4.53 \pm 2.09$ \\
\hline Operative time (min) & $253.6 \pm 96.4$ \\
\hline Blood loss (ml) & $266.6 \pm 248.3$ \\
\hline Blood transfusion (units) & $1.1 \pm 1.02$ \\
\hline Intraoperative $x$-ray dose (mGy) & $3.21 \pm 1.4$ \\
\hline
\end{tabular}

Values are expressed as mean $\pm \mathrm{SD}$, frequency and percentage $(\%)$. RTA, road traffic accident; min, minutes; ml, milliliters; mGy, milliGray.

reduction in 6 cases and 2 cases with poor reduction was recorded (Fig. 2).

Follow up. The average follow-up was 22.5 months (range: 13-33 months). Malunion, loss of reduction or implant loosening were not recorded. Late complications included one case of avascular necrosis of the femoral head (6 months after surgery) and post-traumatic arthritic changes in 5 cases (directed correlated with comminuted posterior wall fracture associated). Among these, 4 patients, which did not require previous plate removal, required total hip arthroplasty and they were not included in the follow-up.

Evaluating the hip joint function, in the remaining group (26 patients), a mean Merle d'Aubigné-Postel score of $13.26 \pm 4.46$ and a mean Harris score of $86.03 \pm 13.37$ were recorded. The clinical outcome was excellent in 17 (65.3\%), good in $6(23 \%)$, fair in $2(7.7 \%)$ and poor in one $(3.8 \%)$ patient. Radiological grading by Matta criteria was excellent in $19(73 \%)$, good in $6(23 \%)$ and fair in one (3.8\%) patient.

\section{Discussion}

Fixation of acetabular fractures which are associated with severely displaced quadrilateral plate and arcuate line involvement are frequently challenging. Initially, stabilization of the quadrilateral plate fractures was based on anterior reconstruction plates through ilioinguinal approach. Letournel, in 1993 (18), first described the combination of anterior plate with screws into the posterior column with good effects, but he could not directly fix the quadrilateral plate fragment. Then, numerous techniques, based on the buttress effect of the plate were described: One-third tubular plate, ' $\mathrm{T}$ ' plate, 'L' plate (19-21).

With the introduction of the modified Stoppa approach, the use an infrapectineal plate to stabilize the quadrilateral plate become more frequent (22).

As the anatomically shaped plates became more and more popular in trauma surgery, they were also introduced in pelvic surgery. It is well known that in pelvic surgery the shape of the plate needs to be as close as possible as the anatomic shape of the bone to achieve good reduction and stabilization. For these reasons, there are two problems that need to be taken into consideration: Significant variation in the size and shape of the pelvic inlet and the use of titanium alloy that could not be shaped intraoperatively. Under these conditions the plate needs to be shaped for each individual patient.

In the effort to use a single plate that offers good stability of both arcuate line fracture and quadrilateral plate, different type of plates were developed. One of these was the omega plate. The plate has a relatively large surface area of $20 \mathrm{~cm}^{2}$ that stabilises the quadrilateral plate and the surrounding area around the arcuate line. In a short study of 15 patients, the authors concluded that the rigidity of the plate provides adequate support for the final reduction by compression and prevents further femoral head protrusion into the lesser pelvis area (23). In the present study, for 26 patients, the plate offered good fixation of different types of acetabular fractures associated with quadrilateral plate fracture and no implant loosening was observed, which may suggest good stability offered by the plate. The clinical and radiological results were similar to those reported by the developers.

Modified Stoppa approach offered us direct visualisation of the quadrilateral plate and therefore easier reduction of the fracture. The possibility of precise and anatomical plate precontouring according to the individual preoperative CT scan has led to significant simplification of the reduction manoeuvres, and easier pelvic inlet reconstruction which finally led to decreased intraoperative irradiation dose, lower blood loss and shortening of the overall operative time.

To simplify the pre-contouring process of the plate, according to the developers, the manufacturer offers the possibility of precise plate contouring based on the patient CT examination (14). This helps during the surgery and is also helpful in the future with the possibility of receiving precontoured titanium plates. 


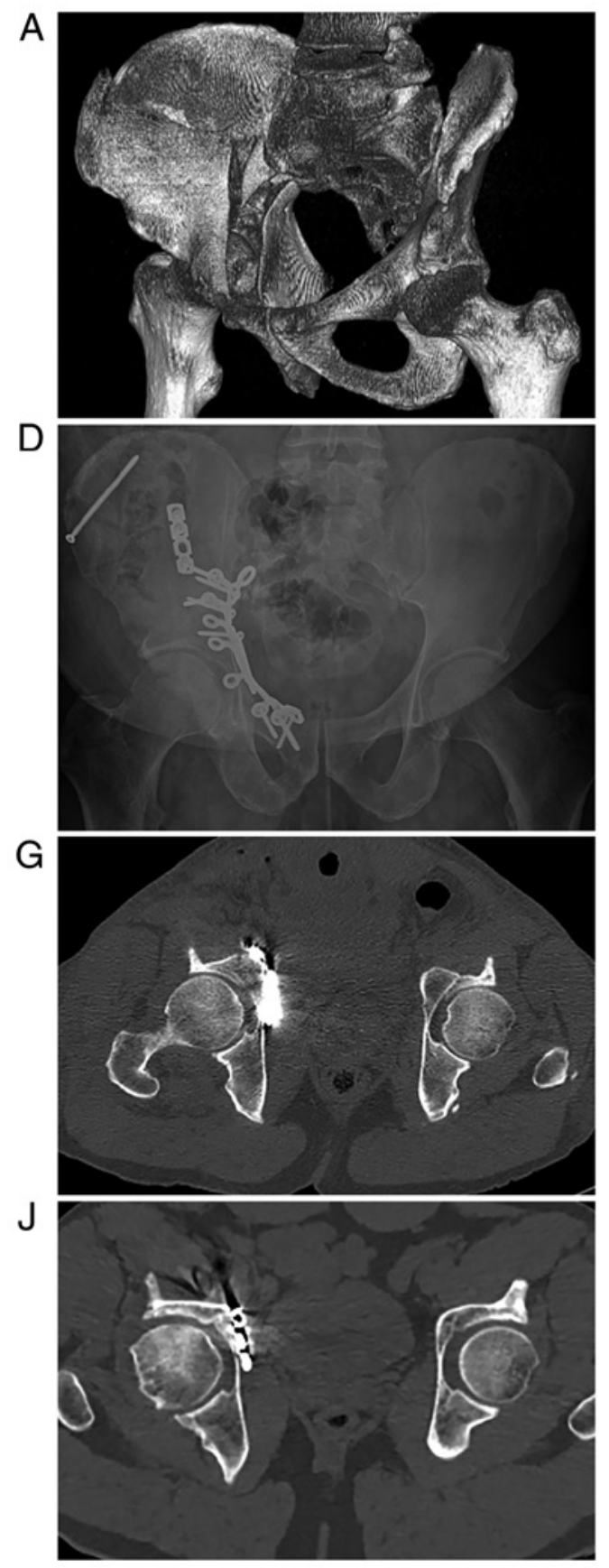

B

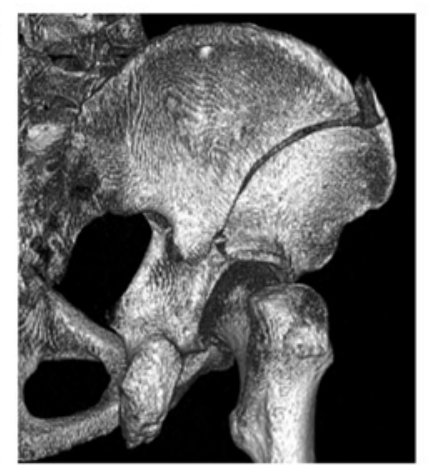

$\mathrm{E}$

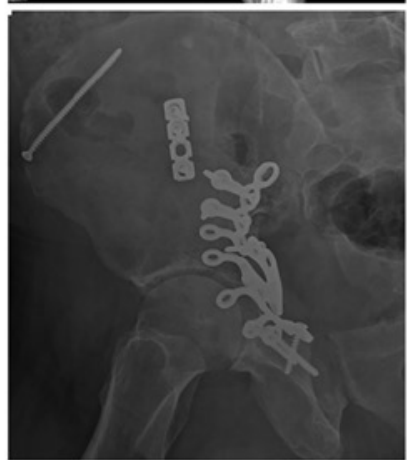

$\mathrm{H}$

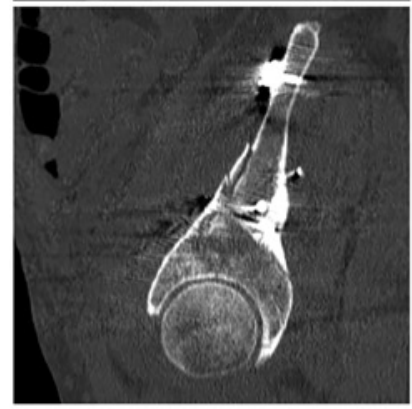

K

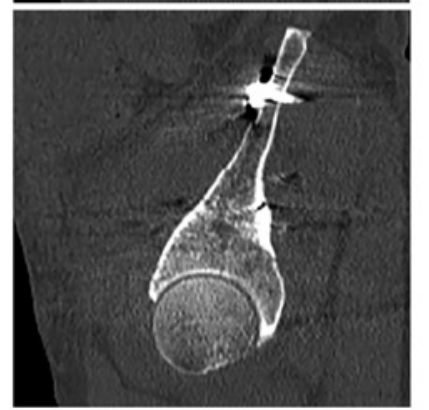

C

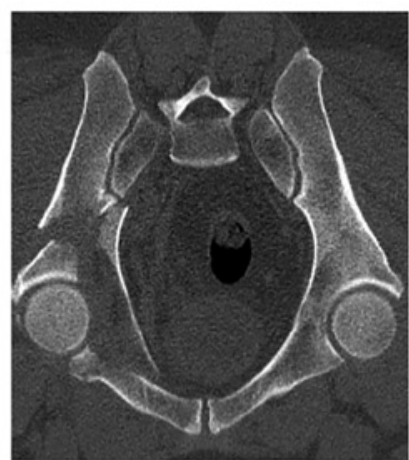

$\mathrm{F}$

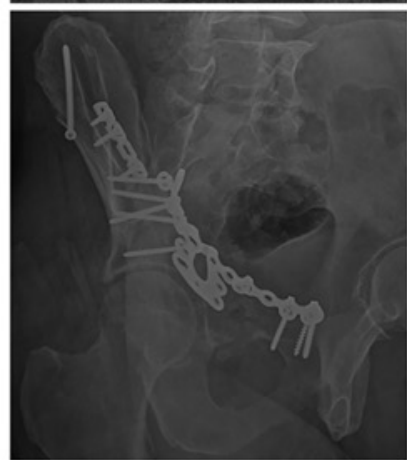

I

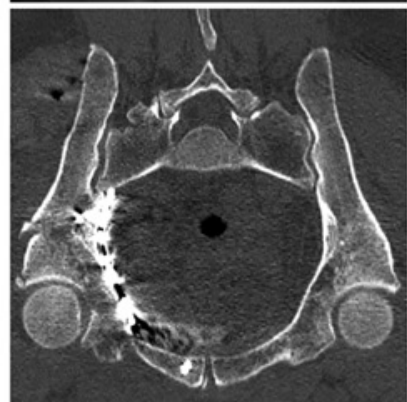

L

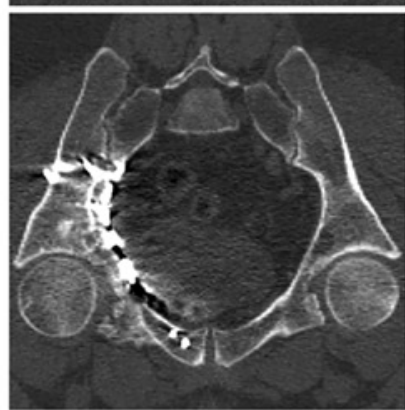

Figure 2. Example of anterior column + posterior hemitransverse acetabular fracture (56-year-old man). (A and B) Preoperative 3D CT scan. (C) Axial CT scan view center over the cranial $1 / 4$ of the femoral head for preoperative plate contouring. Postoperative X-rays [(D) antero-posterior, (E) iliac oblique and (F) obturator oblique views] - internal fixation with omega plate throughout modified Stoppa approach and additional fixation with a reconstruction plate and a screw for the iliac wing (high anterior column) throughout the lateral window. Postoperative CT scan [(G) axial, (H) sagittal and (I) pelvic inlet views] - articular congruence with anatomical reduction of the acetabular surface (Matta 1), realignment of the pelvic arch. Postoperative CT scan after 14 months $[(\mathrm{J})$ axial, (K) sagittal and (L) pelvic inlet views)].

One of the strengths of the present study is that the surgery was performed by the same surgeon and the limitation results from the relatively small number of cases and few studies reported in the literature for comparison.

The omega plate has a number of characteristics that enables primary stability and fixation of the primary acetabular fracture fragments and the quadrilateral plate, possibility of a preoperative anatomically shaped plate, highly resistant to secondary loss of reduction combined with a single anterior, and modified Stoppa approach. Thus, the omega plate represents a viable option for treating acetabular fractures with a very low complication rate and good to excellent results in $89 \%$ of the cases.

\section{Acknowledgements}

Not applicable.

\section{Funding}

No funding was received. 


\section{Availability of data and materials}

The datasets used and/or analyzed during the current study are available from the corresponding author on reasonable request.

\section{Authors' contributions}

$\mathrm{BV}$ and $\mathrm{OA}$ conceived the study and confirm the authenticity of all the raw data. BV, AF and MP analyzed and interpreted the patient data with acetabular fractures. DP and CC searched the literature and wrote the first draft of the manuscript. BV, AF, MP and OA are accountable for all aspects related to the accuracy or integrity of the work and revised the manuscript. All authors read and approved the final manuscript for publication.

\section{Ethics approval and consent to participate}

Informed consent was obtained from all the individual participants included in the study. The study was conducted according to the Helsinki Declaration and was approved by the Ethics Committee of the 'Sf. Spiridon' Emergency Hospital Iasi, Romania.

\section{Patient consent for publication}

Not applicable.

\section{Competing interests}

The authors declare that they have no competing interests.

\section{References}

1. Matta JM: Fractures of the acetabulum: Accuracy of reduction and clinical results in patients managed operatively within three weeks after the injury. J Bone Joint Surg Am 78: 1632-1645, 1996

2. Judet R, Judet J and Letournel E: Fractures of the acetabulum: Classification and surgical approaches for open reduction. Preliminary report. J Bone Joint Surg Am 46: 1615-1646, 1964.

3. Giannoudis PV, Grotz MR, Papakostidis C and Dinopoulos H: Operative treatment of displaced fractures of the acetabulum. A meta-analysis. J Bone Joint Surg Br 87: 2-9, 2005

4. Briffa N, Pearce R, Hill AM, Bircher M: Outcomes of acetabular fracture fixation with ten years' follow-up. J Bone Joint Surg Br 93: 229-236, 2011.

5. Letournel E, Judet R and Elson RA: Surgical approaches to the acetabulum. In: Fractures of the Acetabulum. Elson RA (ed). Springer, Berlin, pp363-397, 1993.

6. Matta JM: Operative treatment of acetabular fractures through the ilioinguinal approach. A 10 -year perspective. Clin Orthop Relat Res: Aug 1, 1994 (Epub ahead of print).
7. Gaheer RS, Rysavy M, Al Khayarin MM and Kumar K: Femoral artery intimal injury following open reduction of an acetabular fracture. Orthopedics 32: 212, 2009.

8. Yamamoto N, Noda T, Saito T, Uehara T, Shimamura Y, Ozaki T: External iliac artery thrombosis following open reduction of acetabular fracture: A case report and literature review. Arch Orthop Trauma Surg 140: 481-485, 2020.

9. Badulescu O V, Filip N, Sirbu P D, Bararu-bojan I, Vladeanu M, Bojan A, Ciocoiu M: Current practices in haemophilic patients undergoing orthopedic surgery-a systematic review. Exp Ther Med 20: 207, 2020.

10. Cole JD and Bolhofner BR: Acetabular fracture fixation via a modified Stoppa limited intrapelvic approach. Description of operative technique and preliminary treatment results. Clin Orthop Relat Res 305: 112-123, 1994.

11. Hirvensalo E, Lindahl J, Kiljunen V: Modified and new approaches for pelvic and acetabular surgery. Injury 38: 431-441, 2007.

12. Andersen RC, O'Toole RV, Nascone JW, Sciadini MF, Frisch HM and Turen CW: Modified stoppa approach for acetabular fractures with anterior and posterior column displacement: Quantification of radiographic reduction and analysis of interobserver variability. J Orthop Trauma 24: 271-278, 2010.

13. Alexa-Stratulat T, Neagu M, Neagu AI, Alexa ID and Ioan BG: Consent for participating in clinical trials - Is it really informed? Dev World Bioeth 18: 299-306, 2018.

14. Taller S, Srám J, Lukáš R, Endrych L and Džupa V: Fixation of acetabular fractures. a novel method of pre-operative Omega plate contouring. Acta Chir Orthop Traumatol Cech 81: 212-220, 2014 (In Czech).

15. Matta JM, Mehne DK and Roffi R: Fractures of the acetabulum. Early results of a prospective study. Clin Orthop Relat Res: 241-250, 1986.

16. D'Aubigne RM and Postel M: Functional results of hip arthroplasty with acrylic prosthesis. J Bone Joint Surg Am 36-A: 451-475, 1954.

17. Harris WH: Traumatic arthritis of the hip after dislocation and acetabular fractures: Treatment by mold arthroplasty. An end-result study using a new method of result evaluation. J Bone Joint Surg Am 51: 737-755, 1969.

18. Letournel E: The treatment of acetabular fractures through the ilioinguinal approach. Clin Orthop Relat Res 292: 62-76, 1993.

19. Wu YD, Cai XH, Liu XM, Zhang HX: Biomechanical analysis of the acetabular buttress-plate: Are complex acetabular fractures in the quadrilateral area stable after treatment with anterior construct plate-1/3 tube buttress plate fixation? Clinics (Sao Paulo) 68: 1028-1033, 2013.

20. Tosounidis TH, Gudipati S, Panteli M, Kanakaris NK and Giannoudis PV: The use of buttress plates in the management of acetabular fractures with quadrilateral plate involvement: Is it still a valid option? Int Orthop 39: 2219-2226, 2015.

21. Peter RE: Open reduction and internal fixation of osteoporotic acetabular fractures through the ilio-inguinal approach: Use of buttress plates to control medial displacement of the quadrilateral surface. Injury 46 (Suppl 1): S2-S7, 2015.

22. Qureshi AA, Archdeacon MT, Jenkins MA, Infante A, DiPasquale $\mathrm{T}$ and Bolhofner BR: Infrapectineal plating for acetabular fractures: A technical adjunct to internal fixation. J Orthop Trauma 18: 175-178, 2004.

23. Šrám J, Taller S, Lukáš R and Endrych L: Use of the Omega plate for stabilisation of acetabular fractures: First experience. Acta Chir Orthop Traumatol Cech 80: 118-124, 2013 (In Czech). 